\title{
gs \\ Harmonic ratcheting for fast acceleration
}

\author{
N. Cook, ${ }^{* \dagger}$ J. M. Brennan, and S. Peggs \\ Collider-Accelerator Department, Brookhaven National Laboratory, Upton, New York 11973, USA
}

(Received 16 February 2014; published 17 April 2014)

\begin{abstract}
A major challenge in the design of rf cavities for the acceleration of medium-energy charged ions is the need to rapidly sweep the radio frequency over a large range. From low-power medical synchrotrons to high-power accelerator driven subcritical reactor systems, and from fixed focus alternating gradient accelerators to rapid cycling synchrotrons, there is a strong need for more efficient, and faster, acceleration of protons and light ions in the semirelativistic range of hundreds of $\mathrm{MeV} / \mathrm{u}$. A conventional way to achieve a large, rapid frequency sweep (perhaps over a range of a factor of 6 ) is to use custom-designed ferriteloaded cavities. Ferrite rings enable the precise tuning of the resonant frequency of a cavity, through the control of the incremental permeability that is possible by introducing a pseudoconstant azimuthal magnetic field. However, rapid changes over large permeability ranges incur anomalous behavior such as the "Q-loss" and "f-dot" loss phenomena that limit performance while requiring high bias currents. Notwithstanding the incomplete understanding of these phenomena, they can be ameliorated by introducing a "harmonic ratcheting" acceleration scheme in which two or more rf cavities take turns accelerating the beam-one turns on when the other turns off, at different harmonics—so that the radio frequency can be constrained to remain in a smaller range. Harmonic ratcheting also has straightforward performance advantages, depending on the particular parameter set at hand. In some typical cases it is possible to halve the length of the cavities, or to double the effective gap voltage, or to double the repetition rate. This paper discusses and quantifies the advantages of harmonic ratcheting in general. Simulation results for the particular case of a rapid cycling medical synchrotron ratcheting from harmonic number 9 to 2 show that stability and performance criteria are met even when realistic engineering details are taken into consideration.
\end{abstract}

DOI: 10.1103/PhysRevSTAB.17.042001

PACS numbers: 29.20.dk, 29.27.Bd, 75.50.Bb, 87.56.bd

\section{INTRODUCTION}

Ions in a synchrotron accelerate over a speed range

$$
\beta_{\min } \leq \beta(t) \leq \beta_{\max }
$$

Isochronous accelerators maintain a constant revolution frequency as particles gain energy through careful adjustment of path length. In principle, the isochronous condition can be maintained by allowing the change in transit time $\Delta T$ between two consecutive cavities to be a multiple of the rf period [1]. This characteristic becomes exceedingly difficult to maintain as particles become relativistic, requiring a nonlinear increase in magnetic fields guiding the trajectory.

Thus, the rf voltage is often varied to maintain performance in a nonisochronous environment. The revolution

\footnotetext{
* corresponding author

ncook@bnl.gov

Also at Department of Physics and Astronomy, Stony Brook University, Stony Brook, NY 11794, USA.
}

Published by the American Physical Society under the terms of the Creative Commons Attribution 3.0 License. Further distribution of this work must maintain attribution to the author $(s)$ and the published article's title, journal citation, and DOI. frequency for an accelerating particle sweeps over the corresponding range

$$
\frac{\beta_{\min } c_{0}}{C} \leq f_{\text {rev }}(t) \leq \frac{\beta_{\max } c_{0}}{C},
$$

where $c_{0}$ is the speed of light and $C$ is the synchrotron circumference. At any instant in the acceleration cycle, the rf system must provide an accelerating voltage of

$$
V_{\mathrm{acc}}(t)=\frac{1}{f_{\text {rev }}(t) Z e} \frac{d K}{d t}
$$

to synchronous ions on every turn, where $Z e$ is the charge on the ion, and $K(t)$ is the periodic kinetic energy ramp. The total gap voltage $V_{g}>V_{\text {acc }}$ and the synchronous phase $\phi_{s}$ also vary periodically, so that an ion with a longitudinal displacement $z$ from the design path experiences an $\mathrm{rf}$ acceleration of

$$
V(z)=V_{g} \sin \left(h \frac{2 \pi z}{C}+\phi_{s}\right) .
$$

The integer harmonic number $h$ relates the radio and revolution frequencies through the relationship 


$$
f_{\text {rf }}=h f_{\text {rev }} .
$$

Despite considerable improvements to rf technology and engineering, conventional acceleration of low $\beta$ charged particles remains expensive and inefficient. Nonetheless, the potential for faster acceleration of low energy ion beams remains rich, with a host of applications proposed to take advantage of rapid cycling synchrotrons (RCSs) or fixed focus alternating gradients [2] [3] [4]. The primary challenge of accelerating such particles remains the wide frequency range requiring robust and flexible tuning regimens. Using speciality ferrites, a broad tuning range can be obtained at the cost of efficiency [5]. In many cases, the ferrite materials suffer dramatic loss effects when pushed to large biasing fields, rapid biasing changes, and large amplitude ac fluxes [6] [7]. These difficulties limit the advancement of fast and efficient accelerators for low energy ion beams.

Several different approaches have been considered as means of improving broadband $\mathrm{rf}$ performance. In lieu of ferrite, magnetic alloy (MA) cavities have been designed and used for high power rapid cycling synchrotrons [8]. Another solution is to chose the energy gain per turn such that cavities are spaced by an integer multiple of the rf period, thus allowing accelerating bunches to skip rf buckets. This is the premise of the "harmonic number jump" scheme, providing a dynamical adjustment of $h$ to extend the viable dynamical range of a fixed-frequency cavity [9] [10]. Alternately, the required rf range can be significantly reduced below the revolution frequency range by decreasing $h$ in steps as the ions accelerate and $f_{\text {rev }}$ increases. This is the motivation and the basic method of harmonic ratcheting.

\section{HARMONIC RATCHETING}

Suppose that two sets of rf cavities take turns accelerating the beam-one turns on when the other turns off, at different radio frequencies-so that the radio frequency is always constrained to remain in the range

$$
f_{\min } \leq f_{r f} \leq f_{\max },
$$

where $f_{\min }$ and $f_{\max }$ are externally determined design parameters. It is possible to make the transition back or forth between harmonic numbers

$$
h=n, \quad \text { and } \quad h=n+\Delta,
$$

where $n$ and $\Delta$ are positive integers, if

$$
\frac{f_{\max }}{n+\Delta}>\frac{f_{\min }}{n}
$$

that is, if

$$
\Delta<n r
$$

where

$$
r \equiv \frac{f_{\max }}{f_{\min }}-1>0
$$

is the "ratcheting parameter." Equation (9) shows that $\Delta$ has a maximum permissible value, which must be greater than 1 if a harmonic transition is to be possible. This is guaranteed for all values of $n$ in the case that $r>1$, when the radio frequency swings by more than a factor of 2 . If the swing ratio is less than 2, then $r<1$ and transition is only possible if

$$
n>\frac{1}{r}, \quad r<1 \text {. }
$$

Many harmonic transitions can take place during one acceleration ramp. Figure 1 shows an example with $r=0.50$, where the revolution frequency increases from 0.61 to $3.35 \mathrm{MHz}$, while the radio frequency is constrained to lie between 5.5 and $8.3 \mathrm{MHz}$. Figure 2 shows the radio frequency and total gap voltage as a function of time for the same ramp.

\section{A. Designing a ratcheting ramp}

One constraint on the initial harmonic number in the ratcheting ramp is imposed by the minimum required bucket length, $t_{\mathrm{inj}}$, to accept particles into a single bucket at injection. This length sets a maximum possible initial harmonic. Moreover, the energy acceptance at injection also scales inversely with $h_{\max }{ }^{2}$. These requirements can be summarized by

$$
h_{\max } \leq \frac{1}{t_{\mathrm{inj}} f_{\mathrm{rev}}(0)}, \quad \text { and } \quad h_{\max }{ }^{2} \leq \frac{2 Q V_{g} \beta^{2} E_{s}}{\pi\left|\eta_{s}\right| \Delta E_{0}{ }^{2}}
$$

Here, $Q=Z e$ is particle charge, $E_{s}$ is synchronous energy, and $\eta_{s}$ is the phase slip factor. As the initial harmonic increases, a corresponding increase in voltage is necessary to keep the acceptance the same.

\section{B. Emittance growth at ratcheting transition}

During the harmonic transition, the voltage on the cavity operating near $f_{\max }$ at harmonic $h=n$ is reduced while the voltage on a second cavity operating at harmonic $h=n+\Delta$ is raised to provide the desired gap voltage at the new harmonic. Assume that this change happens quickly (nonadiabatically) and that both the synchronous phase and total accelerating voltage are smoothly varying and/or fixed when the ratcheting takes place. The accelerating bucket area, $A$, scales with the harmonic number as 


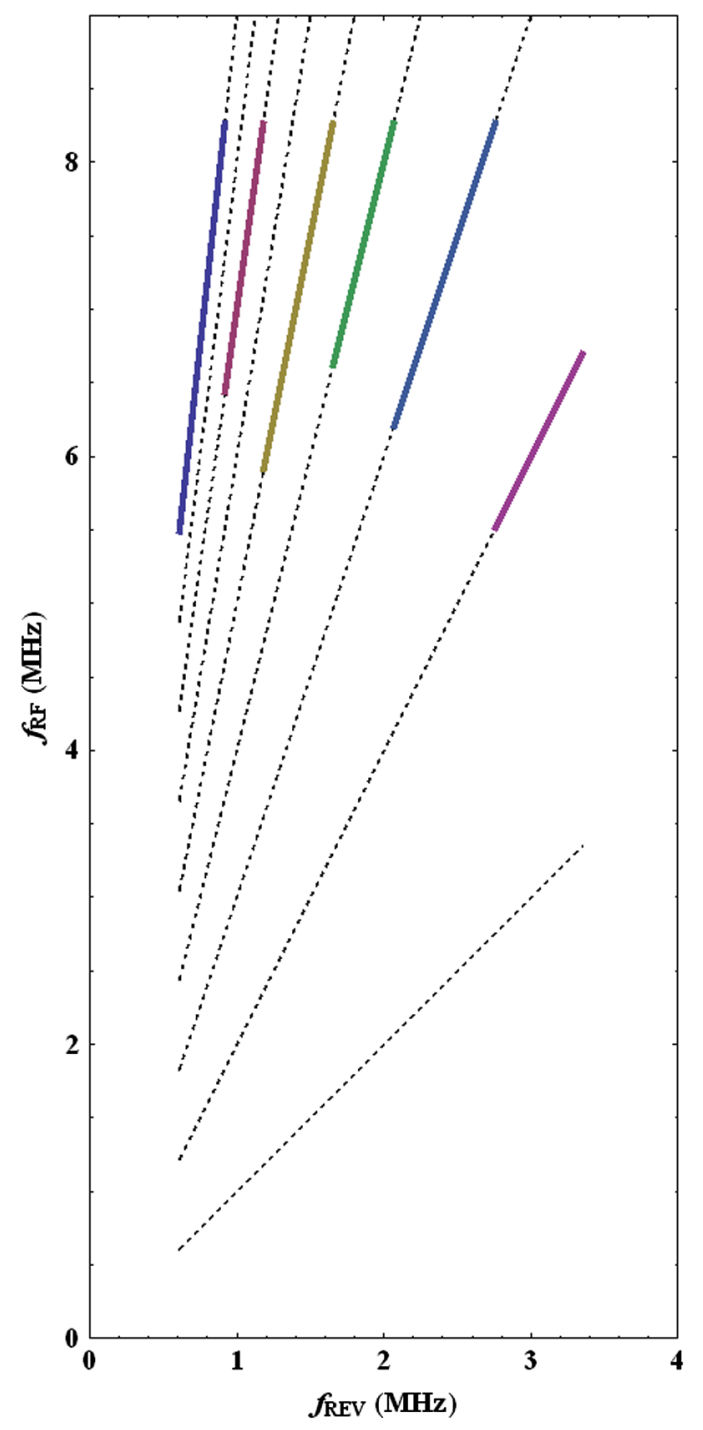

FIG. 1. An example of harmonic ratcheting with $r=0.50$, in the case where the revolution frequency increases from 0.61 to $3.35 \mathrm{MHz}$, while the radio frequency is constrained between 5.5 and 8.25 MHz. Color coded segments show one possible solution-with the harmonic number sequence $h=9,7,5,4,3$, 2 - that maximizes the radio frequency while minimizing the number of ratchets.

$$
A \propto \frac{1}{h^{\frac{3}{2}}}
$$

Assume the ramp undergoes a harmonic change according to Eq. (7), with the harmonic number being reduced from $n+\Delta$ to $n$, and the total voltage does not change appreciably. The bucket area grows according to

$$
A_{f}=\left(\frac{n+\Delta}{n}\right)^{\frac{3}{2}} A_{i}
$$

Because the synchronous phase remains the same, it is only the slope of the voltage curve with respect to particle
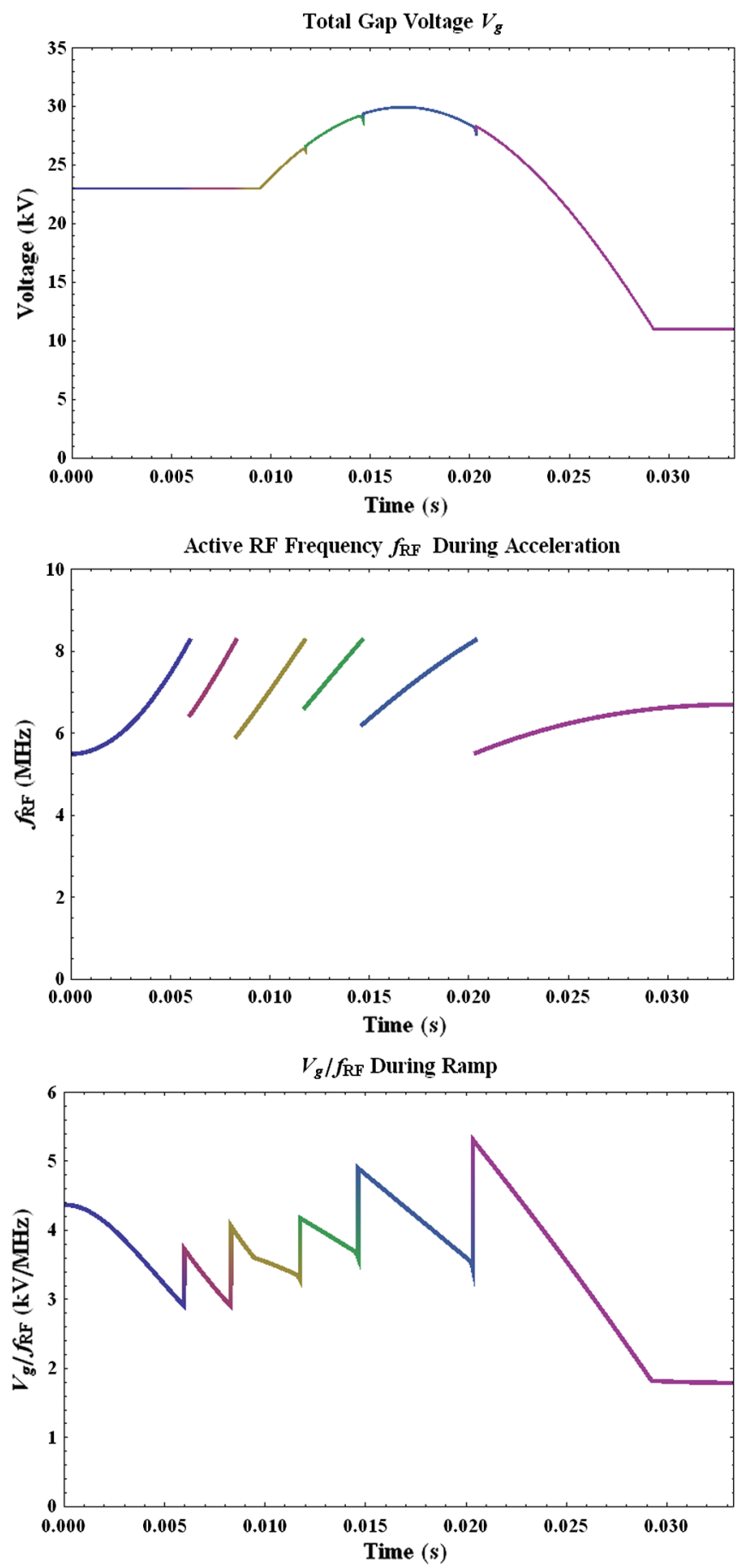

FIG. 2. Active cavity parameters for a $65 \mathrm{~m}$ carbon synchrotron operating at $15 \mathrm{~Hz}$ with ratcheting sequence of $h=9,7,5,4,3,2$. Top: active cavity voltage is shown versus ramp time. Middle: active cavity frequency. Bottom: ratio of cavity voltage to frequency is plotted; this ratio remains fairly consistent throughout the entire acceleration cycle.

position that changes, and not the total magnitude of accelerating voltage. Thus, the transition from a larger to smaller harmonic does not result in a net shift in bunch position but instead relaxes the voltage curve defining the 
stable longitudinal phase space. In order to achieve the same restoring voltage slope, a particle with phase displacement $\theta_{i}$ under the previous harmonic $n+\Delta$ extends to a new phase coordinate

$$
\theta_{f}=\frac{n+\Delta}{n} \theta_{i}
$$

This results in a change in the rms momentum spread, $\sigma$, of

$$
\sigma_{f}^{2}=\left(\frac{n+\Delta}{n}\right) \sigma_{i}^{2}
$$

The 95\% longitudinal emittance of the beam, assumed Gaussian, follows from

$$
S=K\left(\frac{\sigma_{p}}{p_{s}}\right)^{2}
$$

with

$$
K=\frac{3}{h f_{\text {rev }}} \sqrt{\frac{-2 \pi h \eta E_{s}^{3} \beta^{2}}{e V_{g} \cos \phi_{s}}},
$$

where $p_{s}$ is the synchronous particle momentum, $E_{s}$ is the synchronous particle energy, and $e$ is the elementary charge of an electron [11]. Using Eqs. (16) and (18), the new emittance becomes

$$
S_{f}=\left(\frac{n+\Delta}{n}\right)^{\frac{3}{2}} S_{i}
$$

Thus the emittance growth due to the harmonic number transition in Eq. (19) matches the bucket area growth due to the same transition found in Eq. (14). We have

$$
\frac{S_{i}}{A_{i}}=\frac{S_{f}}{A_{f}} .
$$

For slow changes in harmonic number, emittance growth is precisely checked by an increase in bucket area, making the ratcheting process inherently stable. This property can be seen in Fig. 6 for the example ramp in which the transition takes place nonadiabatically.

\section{FERRITE-LOADED CAVITIES}

The resonant frequency of a ferrite-loaded cavity is

$$
f=\frac{1}{2 \pi} \frac{1}{\sqrt{L C}},
$$

where $L$, the inductance of the cavity, is adjustable between minimum and maximum value $L_{\min }$ and $L_{\max }$, corresponding to frequencies $f_{\max }$ and $f_{\min }$, respectively. The minimum inductance that is required is given by solving the equation

$$
\frac{f_{\max }}{f_{\text {min }}}=1+r=\sqrt{\frac{L_{\text {max }}+L_{\text {stray }}}{L_{\text {min }}+L_{\text {stray }}}} .
$$

When $r$ is small, $L_{\min }$ is large, and it is possible to ignore the stray inductance, $L_{\text {stray }}$ (which is usually on the order of several $\mu \mathrm{H}$ ); however, in the case of a large frequency swing the entire range of plausible inductances must be tuned. Figure 3 demonstrates the reliance upon small stray inductance for large $r$ values, using Phillips 4L2 ferrite for tuning. Ratcheting permits larger stray inductances.

Consider a cavity with a total ferrite length of $l$, made of many rings with inner and outer radii $r_{a}$ and $r_{b}$. The inductance is

$$
L=\frac{l}{2 \pi} \mu^{\prime} \ln \frac{r_{b}}{r_{a}},
$$

where the incremental complex permeability of the ferrite is written

$$
\mu=\mu^{\prime}+j \mu^{\prime \prime} .
$$

The component $\mu^{\prime}$ and the inductance of the cavity are tuned by biasing the ferrite with a pseudoconstant azimuthal magnetic field driven by a tuning current. Ignoring the stray inductance, the required dynamic range of $L$ and $\mu^{\prime}$ is

$$
\frac{L_{\min }}{L_{\max }}=\frac{\mu_{\min }^{\prime}}{\mu_{\max }^{\prime}}=\frac{1}{(1+r)^{2}} .
$$

The voltage across the gap of a cavity is

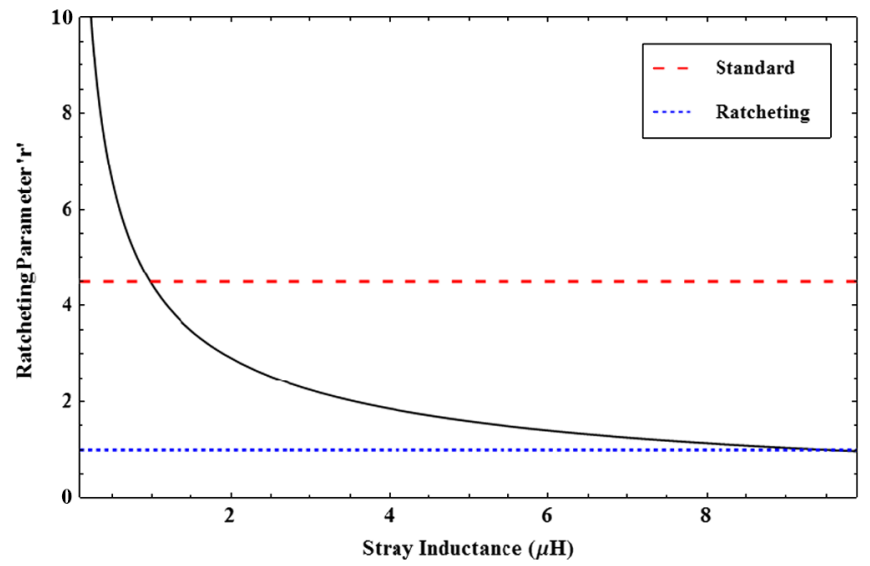

FIG. 3. The achievable ratcheting parameter $r$ is plotted for the ferrite Phillips 4L2 using fitting data collected at BNL [12]. The plot demonstrates that, for a fixed capacitance, a low stray inductance is needed to achieve a high frequency ratio. However, for low values, $\frac{f_{\max }}{f_{\min }}<2$, inductances of several $\mu \mathrm{H}$ are acceptable. 


$$
V_{\mathrm{gap}}=2 \pi f B_{\max } l r_{a} \ln \frac{r_{b}}{r_{a}} \sim f l,
$$

where $B_{\max } \approx 0.01 \mathrm{~T}$ is the maximum incremental $\mathrm{rf}$ magnetic field that is allowed at the inner radius of the ferrite rings, oscillating at the frequency $f$.

For a fixed ferrite ring geometry $\left(r_{a}\right.$ and $\left.r_{b}\right)$, and a fixed maximum field $B_{\max }$, the gap voltage scales with ferrite length and cavity frequency. This shows that the ferrite length $l$ can be reduced if the radio frequency in the cavity is increased. By inflating cavity frequency, harmonic ratcheting allows a given acceleration waveform to be achieved with shorter ferrite-and shorter cavities.

The total length of ferrite required with a ratcheting parameter $r$, relative to a nonratcheting scheme in which ions are accelerated over the full dynamic range

$$
D=\frac{\beta_{\max }}{\beta_{\min }}=\frac{f_{\text {rev, max }}}{f_{\text {rev, min }}},
$$

is given approximately by

$$
\frac{l_{\text {ratchet }}}{l_{\text {nonratchet }}}=\frac{f_{\text {min,nonratchet }}}{f_{\text {min,ratchet }}} \approx 2 \frac{1+r}{D},
$$

where the factor of 2 recognizes that only half of the cavities are active at any one time. For example, Fig. 1 illustrates acceleration over a dynamic range of $D=5.5$, using a ratcheting parameter of $r=0.50$. In this case ratcheting (ideally) makes it possible to decrease the total length of ferrite by a factor of 0.55 . Conversely, the gap voltage can be almost doubled for a fixed amount of $\mathrm{rf}$ cavity real estate, in turn almost doubling the potential repetition rate of a rapid cycling synchrotron.

\section{EXAMPLE: A RAPID CYCLING MEDICAL SYNCHROTRON}

Consider a rapid cycling synchrotron for acceleration of $\mathrm{C}^{6+}$ ions for radiation therapy applications, with a $15 \mathrm{~Hz}$ repetition rate and a circumference of $C=\sim 65 \mathrm{~m}$. Ions are accelerated through a range 8 to $400 \mathrm{MeV} / u$, with a nominal bunch charge of $10 \mathrm{pA}$.

Once the initial harmonic number is determined, the initial radio frequency is set at $f_{r f}(0)=h_{0} \beta c / C$. The frequency ceiling can then be chosen. In this example, we choose a ratcheting parameter of $r=0.5$ via a final harmonic transition of $h=3$ to $h=2$. The resultant frequency swing is shown in Fig. 2, with the harmonic sequence shown in Fig. 4.

When the frequency ceiling is reached after acceleration at one particular harmonic, rf power is transferred to the second cavity system at a lower harmonic number. Each time the harmonic number is reduced, the radio frequency is reduced by a factor $\frac{h_{n+\Delta}}{h_{n}}$ where $h_{n}$ is the new harmonic, and $h_{n+\Delta}$ is the previous value. The maximum frequency

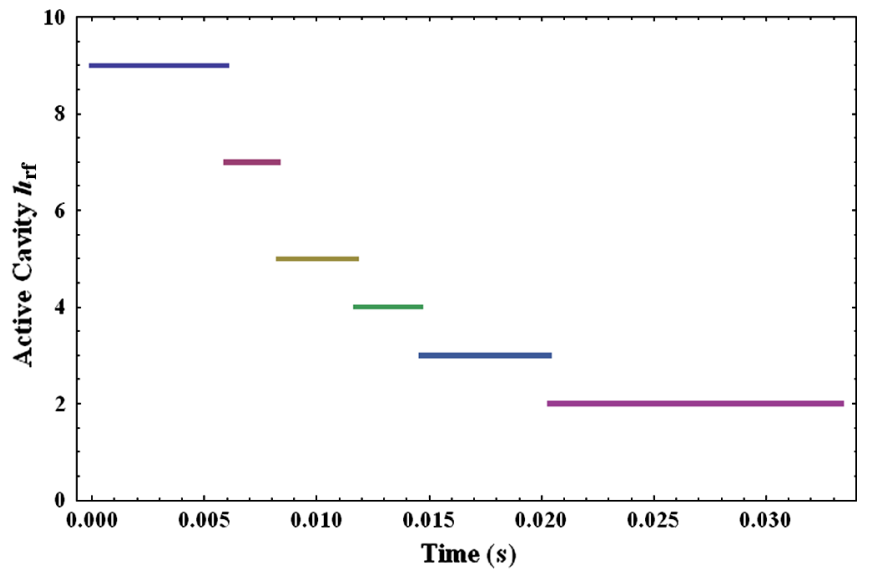

FIG. 4. Active cavity harmonic for the $65 \mathrm{~m}$ carbon synchrotron harmonic ratcheting ramp. The cavity begins at $h=9$ and ratchets in sequence through $h=7,5,4,3,2$.

swing during the ramp is determined by the maximum value of this ratio, or equivalently the ratcheting parameter $r$. For example, if the largest transition in a particular ratcheting scheme is from $h=3$ to $h=2$, then the ratcheting parameter is $r=0.5$, and that particular ramp is limited to a $50 \%$ frequency swing.

Figure 5 shows the output voltage needed for each cavity system in the example ratcheting ramp. The transfer of power in a harmonic transition to the inactive cavity cannot be performed instantaneously. The voltage across the cavity must be raised over many turns of the accelerator. This transition may be on the order of $100 \mu$ s depending upon the voltage level and frequency of the cavity. For a fixed total gap voltage, a change in $h$ constitutes a change in the synchronous phase $\phi_{s}$ of the system according to $\phi_{s, f}=\phi_{s, i} \frac{n}{n+\Delta}$. If the phase is changed too abruptly, the

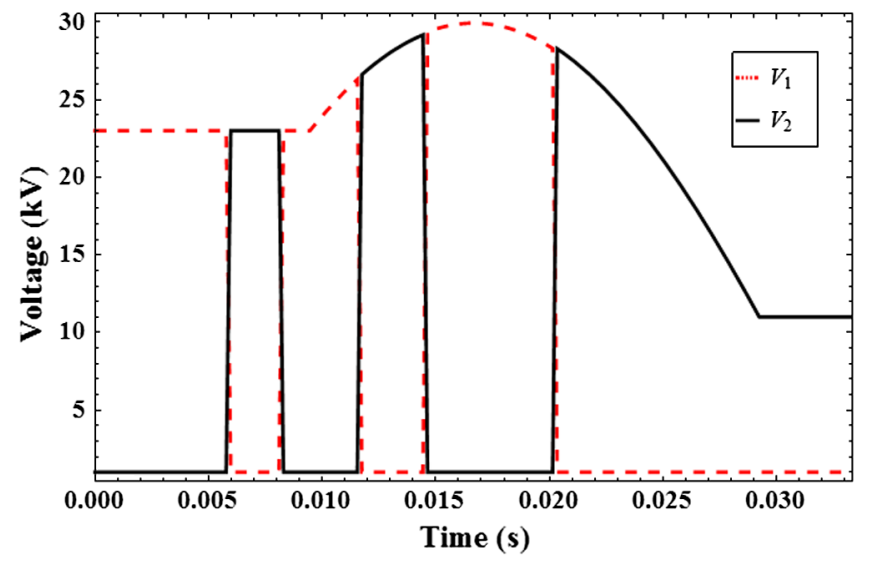

FIG. 5. Output voltage for each cavity system is depicted for a test ratcheting sequence. A ramping time of $100 \mu \mathrm{s}$ is chosen for the transition periods, and total voltages are calculated to meet the constraint $\phi_{s} \leq 33^{\circ}$. A minimum voltage of $1 \mathrm{kV}$ is assumed in order to allow rf tuning and phase synchronization for the "offline" cavities. 


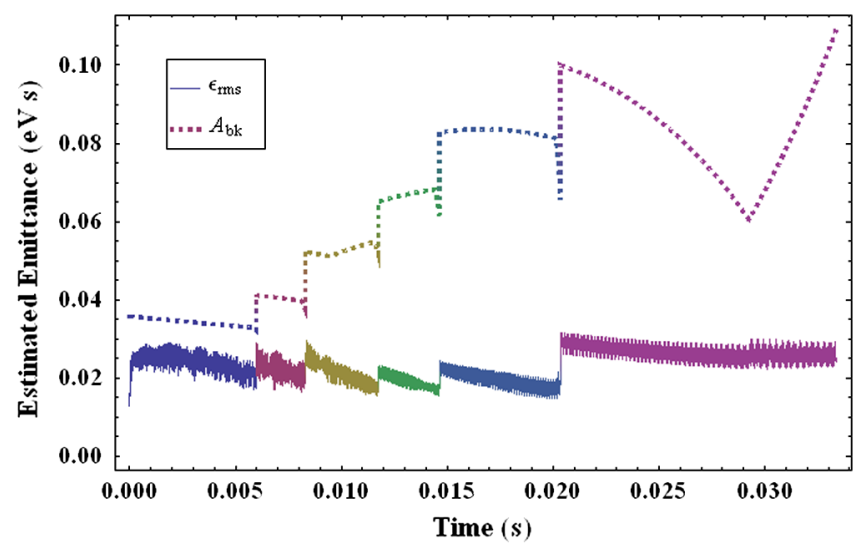

FIG. 6. Root mean square bunch emittance, $\epsilon_{\text {rms }}$, and bucket-area, $A_{\mathrm{bk}}$, are shown for a simulated ramp with carbon ions and a fixed voltage profile. The emittance spikes correspond to the harmonic transitions inducing a quick change in synchronous phase.

bunch center will oscillate about the new value of $\phi_{s}$, and the bunch will filament, causing strands of particles to separate outward from the edge of the bunch and increasing emittance. Assume the transfer of voltage takes place over some time period $\tau_{r}$. A particle will make

$$
n_{t}=\beta c \tau_{r} / C
$$

turns in that time. If $\tau_{r}=100 \mu s$, a $\beta=0.1$ particle makes 46 turns of a $65 \mathrm{~m}$ synchrotron, thus smoothing the dynamics present during the transition period.

An alternative to the fixed voltage profile is to vary the voltage in order to produce a fixed synchronous phase over the course of acceleration. Maintaining a fixed phase reduces emittance growth and bunch filamentation, while reducing power requirements on the rf system. Detailed feedforward and diagnostic systems are required in order to maintain the proper voltage. The emittance evolution for our example synchrotron can be seen in Fig. 6 .

Using the harmonic sequence discussed above, Table I reports an estimate of the new acceleration parameters for the $15 \mathrm{~Hz} \mathrm{C}^{6+}$ acceleration scheme, using Phillips 4L2 and Ferroxcube $8 \mathrm{C} 12$ ferrites [12]. The results underscore the significant reduction in magnetic field, power density, and the extreme decline in the bias field required to tune the ferrite across the smaller relative frequency range.

\section{A. Anomalous effects}

Use of a harmonic ratcheting scheme may also reduce losses in the ferrites due to anomalous effects. At sustained high fields, ferrites demonstrate what is known as the highloss or Q-loss effect (QLE). This can result in reductions of $15 \%-20 \%$ of maximum field. High bias fields can increase this effect by reducing the threshold of Q-loss onset. However, it has been documented that at high bias rates, the threshold for this effect is significantly raised [6]. Given these considerations, a ratcheting scheme should mitigate the QLE by reducing bias fields and maintaining a high rate of change of bias field. High bias field rates may induce a dynamic loss effect in the ferrite. These losses may reduce a cavity's Q value by up to $40 \%$. Unlike the QLE, this effect appears at varying levels of $\mathrm{rf}$ excitation and is more dependent on the temperature and duration of the biasing. By decreasing the time spent biasing before reaching resonance at high fields, the effect can be reduced considerably. It must be stressed that these effects are still poorly understood on a theoretical level, and their effects can vary considerably for different materials.

\section{B. Magnetic alloy cavities}

An alternative to ferrite-loaded cavities is made possible by the use of magnetic alloys, which feature high permeability values, resilience under high magnetic fields, and large inductances. These properties allow MA cavities to achieve low $Q$ values, meaning they can support fast, broadband acceleration using feedforward systems to achieve the desired frequency band operation, as seen in the J-PARC $3 \mathrm{GeV}$ RCS [13]. This low $Q$ value combined with the robust nature of the material allows MA cavities to achieve high gradients, but at the cost of high power requirements, especially at low frequencies wherein the $\mu Q f$ product can be orders of magnitude lower than in ferrite cavities [8]. Despite the resilience of MA cores to

TABLE I. Summary of Physical Parameters and Requirements for $\mathrm{rf}$ Cavity as well as power numbers, assuming $L_{\text {stray }}=0.5 \mu \mathrm{H}$, for $C^{6+}$ acceleration at $15 \mathrm{~Hz}$. We assume the use of two rf cavities with the same geometry, $\mu Q f$ values, and biasing scheme, but either Phillips 4L2 or Ferroxcube 8C12 ferrite. Both ratcheting and nonratcheting ramps are considered for each ferrite.

\begin{tabular}{lccccc}
\hline \hline Cavity parameter & & 4L2 & 4L2 ratcheting & 8C12 & 8C12 ratcheting \\
\hline Harmonic sequence & & $h=1$ & $h=9,7,5,4,3,2$ & $h=1$ & $h=9,7,5,4,3,2$ \\
Radio frequency & {$[\mathrm{MHz}]$} & $0.65-3.57$ & $5.5-8.25$ & $0.65-3.57$ & $5.5-8.25$ \\
Injection bunch length & {$[\mathrm{ns}]$} & 500 & 60 & 500 & 60 \\
Maximum B-field & {$[\mathrm{mT}]$} & 1.33 & 0.96 & 1.33 & 0.96 \\
Maximum gap voltage & {$[\mathrm{kV}]$} & 27.5 & 30 & 27.5 & 30 \\
Max cavity power loss & {$[\mathrm{kW}]$} & 8.4 & 6.7 & 8.4 & 6.7 \\
Permeability range, $\mu_{r}$ & & 4.93 to 200 & 86.9 to 200 & 24.8 to 200 & 86.9 to 200 \\
Maximum bias H & {$[\mathrm{A} / \mathrm{m}]$} & 4485 & 342 & 968 & 320 \\
\hline \hline
\end{tabular}


high fields and temperatures, thermal stress can result in impedance drops and subsequent core damage. The socalled "core buckling" phenomenon requires proactive maintenance schedules requiring the replacement of two sets of cores each year at J-PARC [14]. Recently, such incidents have been found to be reducible by changing the epoxy resin used in core construction. In all, MA cavities provide a number of advantages for high frequency, high gradient operation for RCSs at the expense of power loss and maintenance costs. These tradeoffs are directly correlated to cavity performance, and as such a ratcheting approach would only reduce costs through a similar reduction in performance. Use of MA cavities, as with ratcheting, is useful for particular classes of problems, but they do not complement one another.

\section{CONCLUSION}

In conclusion, a new scheme for accelerating semirelativistic ions quickly and over a large revolution frequency range is presented. The harmonic ratcheting approach combines both permeability change, i.e. frequency change, with harmonic number change, resulting in a viable approach to dynamic broadband rf acceleration. By adjusting the harmonic number, tailored to the desired acceleration cycle, a significant reduction in the radio frequency range is obtained, easing the requirements of tuning currents on the ferrite cavity. Moreover, the subsequent increase in frequency reduces the magnetic flux requirements in the cavity, allowing higher accelerating voltages to be obtained at fixed ferrite length while reducing cavity losses. For reasonable engineering requirements, a two-fold reduction in cavity length, or equivalently a two-fold increase in machine repetition rate, can be achieved for the exact same beam parameters. This approach is widely generalizable, and can be extended to a variety of different acceleration cycles with similar, and in some cases greater, benefits. Harmonic ratcheting is particularly suited to low intensity ion beams that do not require filling the circumference of the ring, but which need fast acceleration across a broad range of speeds. Anomalous effects within the ferrite remain a concern for all ferrite-tuned cavities, but a ratcheting approach seeks to minimize these effects by reducing load on the material. Application of inefficient broadband rf has limited the progress of accelerators with large dynamic ranges in energy; harmonic ratcheting is an important innovation in mitigating this problem.

\section{ACKNOWLEDGMENTS}

We thank D. Trbojevic for many encouraging discussions on this topic. We would also like to acknowledge S. Polizzo and A. Zaltsman for lending their expertise in ferrite materials to provide critical feedback. This work was supported by Brookhaven Science Associates, LLC under Contract No. DE-AC02-98CH10886 with the U.S. Department of Energy.

[1] A. A. Kolomenskii, Sov. Phys. Tech. Phys. 5, 1278 (1961) [A. A. Kolomenskii, Zh. Tekh. Fiz. 30, 1347 (1960)].

[2] K. J. Peach et al., Phys. Rev. ST Accel. Beams 16. 030101 (2013).

[3] S. Peggs, T. Satogata, and J. Flanz, in Proceedings of the 22nd Particle Accelerator Conference, PAC-2007, Albuquerque, NM (IEEE, New York, 2007), pp. 115119.

[4] R. C. Arnold and J. Meyer-ter-Vehn, Rep. Prog. Phys. 50, 559 (1987).

[5] J. M. Brennan, in Handbook of Accelerator Physics and Engineering, edited by A. W. Chao and M. Tigner (World Scientific, Singapore, 1999), pp. 5702.

[6] K. Kaspar, H. G. König, and T. Winnefeld, in Proceedings of the 9th European Particle Accelerator Conference, Lucerne, 2004 (EPS-AG, Lucerne, 2004), pp. 985-987, TUPKF013.

[7] J. E. Griffin and G. Nicholls, IEEE Trans. Nucl. Sci. 26, 3965 (1979).

[8] C. Ohmori et al., Phys. Rev. ST Accel. Beams 16, 112002 (2013).

[9] A. G. Ruggiero, Phys. Rev. ST Accel. Beams 9, 100101 (2006).

[10] T. Planche, J.-B. Lagrange, E. Yamakawa, T. Uesugi, Y. Kuriyama, K. Okabe, Y. Ishi, and Y. Mori, Nucl. Instrum. Methods Phys. Res., Sect. A 632, 7 (2011).

[11] D. A. Edwards and M. J. Syphers, An Introduction to the Physics of High Energy Accelerators (J. Wiley \& Sons, New York, 1993).

[12] Y. Zhao, BNL Informal Report No. CAP-341-RF-R, 2002.

[13] F. Tamura et al., Phys. Rev. ST Accel. Beams 14, 051004 (2011).

[14] M. Nomura et al., Nucl. Instrum. Methods Phys. Res., Sect. A 623, 903 (2010). 